\title{
Editorial
}

\section{Fundamentalismus und neue Religiosität}

I.

Der Fundamentalismus und die in aller Welt neuerwachenden religiösen Bewegungen werden im allgemeinen als Widerstand gegen die Zumutungen einer von Europa ausgehenden Zivilisation, die Traditionen und Glaubenssysteme zersetzt, verstanden. Man verwendet den Begriff $»$ Fundamentalismus $\ll$ in der Regel als globale Bezeichnung für außereuropäische, insbesondere in islamischen Ländern auftretende, defensive Protestbewegungen, die mit religösem Fanatismus an der Konservierung patriarchaler Hierarchien und Fámilienstrukturen arbeiten. Die Ziele dieser Bewegungen richten sich nicht nur ideell gegen die Werte westlicher Aufklärung, sondern sind auch mit demokratischen Formen von Politik, mit religiöser Toleranz und subjektiver Freiheit unvereinbar: Die globalen Prozesse der Differenzierung von Religion und Recht, Politik und Wirtschaft, der Auflösung traditionaler Vergemeinschaftungen und der Entwicklung einer autonomen Individualität können nur mit despotischer Gewalt und Staatsterror zurückgedreht werden.

Diese Interpretation des Fundamentalismus kann sich auf eine lange Tradition berufen, in der die Ausbreitung des industriellen Kapitalismus und der westlichen Kultur als Geschichte des Fortschritts und der Enttraditionalisierung gefeiert und jeder Widerstand als hinterwäldlerische Regression eingestuft wurde. Die Folie, auf der der Kon- trast zwischen der modernen westlichen Zivilisation und den fundamentalistischen Gegenbewegungen seine volle Schärfe erhält, wurde aber nicht nur von der Soziologie, sondern auch vom Marxismus aufgespannt.

Der Marxismus feierte nicht allein den technischen Fortschritt des aufsteigenden Kapitalismus, sondern auch seine kulturrevolutionäre Rolle. Die Durchsetzung universeller Verkehrsverhältnisse löse alte lokale Traditionen und nationale Selbstgenügsamkeiten auf; sie reduziere sentimentale familiäre Bande und persönliche Abhängigkeitsverhältnisse auf ihren nackt kalkulierbaren Geldausdruck; traditionales Bewußtsein und Religion »verdampften«: die »ununterbrochene Erschütterung aller gesellschaftlichen Zustände sorgt für ewige Unsicherheit und Bewegung«. Marx und Engels zögerten nicht, der revolutionären Bourgeoisie im Kommunistischen Manifest zu konzedieren, daß sie durch die »unendlich erleichterte Kommunikation alle, auch die barbarischsten Nationen in die Zivilisation « gerissen und den »hartnäckigsten Fremdenhaß der Barbaren zur Kapitulation gezwungen« habe. Max Weber, der erste soziologische Kritiker des Marxismus, hat die Antithese von Tradition und Moderne zu einer Universalgeschichte verschärft, die dem westlichen Rationalismus sowohl Universalität als auch Einzigartikeit bescheinigt. Allein die westliche Kultur habe eine experimentelle Wissenschaft, ein systematisierungsfähiges Recht und 
einen rational organisierten Kapitalismus hervorgebracht. Antikapitalistischer Protest schrumpft bei Weber auf traditionalistisches Gebaren und ethische Empfindungen. Denn nicht der Kapitalismus, sondern die seinen Aufstieg fördernde Religion trage den Keim ibres Untergangs in sich: aus dem sich selbst reproduzierenden Kapitalismus sei der Geist des Protestantismus verschwunden. Religöses Bewußtsein sei nur noch um den Preis eines »Opfers des Intellekts« oder als Selbstbetrug zu haben. Die Gesellschaftanalysen von Marx und Weber blieben freilich in entscheidender Hinsicht ambivalent. Warenwelt und "Selbstbewegung « des Kapitals erzeugen Marx zufolge eigene Phantasmagorien: vom Warenfetisch bis zu einer regelrechten "Religion des Alltagslebens «, in der alles verkehrt erscheint. Weber war vorsichtig genug, in Wissenschaft als Beruf einzuräumen, daß die »Entzauberung der Welt« womöglich nur auf dem "Glauben « an ihre technische Beherrschbarkeit basiere. Rationalisierung bewirke "ganz und gar nicht eine Universalisierung des Wissens um die Bedingtheiten und Zusammenhänge des Gemeinschaftshandelns ... sondern meist das gerade Gegenteil « (Über einige Kategorien der verstehenden Soziologie).

Erst im soziologischen Mainstream, der Marx bestenfalls vor dem Hintergrund des eigenen Ansatzes »rekonstruiert《 und Webers Religionssoziologie zu einer evolutionären Logik der Modernisierung stilisiert, wurde die Eindeutigkeit erreicht, die der gegenwärtigen Thematisierung des Fundamentalismus entspricht. Dieser Mainstream begreift die Globalisierung des westlichen Modells weniger als faktische Durchsetzung des industriellen Kapitalismus, der außereuropåischen Gesellschaften mit überlegener Technologie und Waffengewalt aufgezwungen wurde, denn als kulturellen Vorgang, der von normativer Überlegenheit zeugt. In diesem Sinn interpretierte Talcott Parsons den Fundamentalismus als religiös motivierten, gesellschaftlichen Konservatismus, der sich gegen die den modernen Gesellschaften zugrundeliegende "Wertverallgemeinerung «, gegen die Ausbildung eines universalen Moralkonsenses, sperre. Damit war ein gemeinsamer Nenner gefunden, auf den Parsons den südafrikanischen Rassismus, faschistische Bewegungen, orthodoxe Kommunisten und 'gewisse Teile' der Neuen Linken brachte. Ganz konsequent wird auf dieser Linie der Untergang des Sozialismus als jüngster Sieg der Moderne über den Traditionalismus der Arbeiterbewegung und den kommunistischen Zentralismus ausgelegt.

Bereits seit einiger Zeit werden auch radikale Ökologen und unbelehrbare Kapitalismuskritiker als fundamentalistische Feinde von Fortschritt und Marktwirtschaft bezeichnet. Ein so verallgemeinerter Begriff von $\gg$ Fundamentalismus « ist anscheinend flexibel genug, um die Leerstelle zu füllen, die der verlorengegangene Antagonist des Kalten Krieges hinterlassen hat. Aufgrund seiner Frontstellung gegen "Verwestlichung « erscheint insbesondere der islamische Fundamentalismus als eine $» \mathrm{Ge}-$ fahr«, die dem Kommunismus in nichts nachsteht, zumal er jetzt auch in den südlichen Nachfolgerepubliken der früheren Sowjetunion, den nordafrikanischen Nachbarregionen Europas wie auch unter den in Westeuropa lebenden Arbeitsmigranten Fuß zu fassen droht. Wendige Köpfe haben die Signale verstanden. Nach dem Ende des Kalten 
Kriegs bläst das Feuilleton zum »Kulturkampf« gegen modernitätsfeindliche Fundamentalisten aller Art, von Atomkraftgegnern bis zu Islamisten.

\section{II.}

Nicht das erstaunliche Vordringen fundamentalistischer Tendenzen und der überraschende Erfolg religiöser Wiedererweckungsversuche seit den späten 70er Jahren sind kontrovers - obwohl diese beiden Entwicklungen die naive Annahme, eine fortschreitende Modernisierung würde Traditionsbestände irreversibel aufzehren, empfindlich erschüttern sollte. Wer wollte bestreiten, daß die Einführung islamischen Rechts im Iran, im Sudan und in Pakistan mit brutaler Repression und der Restauration patriarchaler Gewalt einhergeht? Und es ist auch nicht zu übersehen, daß die Relativierung individueller Menschenrechte im Namen althergebrachter Traditionen allzuoft eine bequeme Rechtfertigung lokaler Machtinteressen ist. Umstritten ist jedoch die Erklärung dieser Entwicklungen, und fatal die Wendung des Fundamentalismusvorwurfs in einen politischen Kampfbegriff.

Durch einen dermaßen überstrapazierten Fundamentalismusbegriff werden Auseinandersetzungen, die aus sozialen Umschichtungen, ökonomischen Verwerfungen und uneingelösten Entwicklungsversprechen resultieren, in letztlich kulturelle oder religiöse Differenzen umgedeutet. Es wäre allerdings auch unzureichend, den Vormarsch fundamentalistischer Bewegungen in den islamischen Ländern als Defensivreaktionen von Gesellschaften zu deuten, die den Druck einer weitergehenden Modernisierung bereits in sich austragen.

Fundamentalismus läßt sich weder auf antiwestliche »Defensivkulturen« noch auf die Beharrungskraft vormoderner Traditionen reduzieren. Der »Westen« selbst hat zum Erstarken etwa des islamischen Fundamentalismus beigetragen, nicht nur durch seine koloniale Vergangenheit, durch fehlgeschlagene Industrialisierungprojekte und enttäuschte Modernisierungsversprechen, sondern auch ganz handfest durch den taktischen Umgang mit innerarabischen Konflikten, die hemmungslose Aufrüstung des Nahen Ostens und die Finanzierung militanter fundamentalistischer Gruppen, die wie in Afghanistan ins geostrategische Kalkül paßten (siehe dazu den Beitrag von Shlomo Avineri).

Werden Tradition und Moderne einander dichotom gegenübergestellt und Fundamentalismus und neue Religiosität einzig einer gegen Substanzverlust kämpfenden Tradition zugerechnet, so werden gerade auch die fundamentalistischen Potentiale, die in der Moderne angelegt sind, übersehen. Bereits die neuzeitliche Expansion der europäischen Mächte war in eine überirdische Verkündungsaura getaucht, und sie wäre ohne ständige religöse Rückversicherung wohl nicht möglich gewesen. Die erste Aufteilung der Welt zwischen Spanien und Portugal wurde von päpstlicher Hand und im Namen der »wahren Religion« vollzogen. Die Aufklärung brachte nicht nur die Kritik von Religion und Aberglauben hervor, sondern mit der Französischen Revolution auch einen »Kult der Vernunft«, dessen Sonnenmythos von Paris aus die Menschheit als ganze erleuchten sollte. Das Ausgreifen des westlichen Zivilisationsmodells auf Nordamerika und darüber hinaus war von dem missionarischen Bewußtsein begleitet, die 》Gemeinschaft Gottes auf Erden« zu ver- 
wirklichen. So ist es kein Zufall, daß der "Fundamentalismus« im ursprünglichen Sinn des Wortes am Anfang dieses Jahrhunderts als protestantische Sektenbewegung in den USA entstand. Und schließlich wurde auch der paranoide Antikommunismus in der von Parsons so bezeichneten »amerikanischen Führungsgesellschaft« der Moderne von der manichäischen Gegenüberstellung von Gut und Böse gespeist und von einem Sendungsbewußtsein getragen, das dem der Kreuzzüge in nichts nachsteht.

\section{III.}

Das vorliegende Heft thematisiert daher weniger die der europäischen Kultur von außen drohende »Gefahr«, als die Fundamentalismen, die in den Kernbereichen der sokzidentalen Rationalisierung« reproduziert werden.

Gerade die Moderne bringt, wie Wolfgang Schmidt hervorhebt, eine eigene Sehnsucht nach Identität hervor, die auch in Europa ihre Erfüllung in verschiedenen »Fundamentalismen « nationaler, ethnischer oder religiöser Gestalt sucht. Auf welch unterschiedliche Weise und zu welch unterschiedlichen Zwecken »kollektive Identität « in diesem Jahrhundert thematisiert wurde, demonstriert Lutz Niethammer in seinem Beitrag.

Rudolf Thiessen entfaltet in seinem von Walter Benjamin ausgehenden Beitrag einen Zusammenhang, welcher der klassischen Religionskritik und auch Max Webers Religionssoziologie entgangen ist, in der Marxschen Gesellschaftsanalyse gleichwohl präsent war. Die durchschlagende Wirkung des modernen Kapitalismus hat nicht zu einer Säkularisierung des Protestantismus geführt, sondern generiert aus sich heraus ein religiöses Potential. Besonders deutlich zeigt sich dieses Potential in den USA. Peter Lohaus unterstreicht in seiner Besprechung von Martin Riesebrodts vergleichender Fundamentalismusstudie, wie tief der protestantische Fundamentalismus in die US-amerikanische Geschichte eingewoben ist und da $B$ er auch gegenwärtig in bestimmten Konstellationen eine durchaus mehrheitsfähige Position abgeben kann. Letzteres legt auch Orlando Pattersons Beitrag über den neuen Puritanismus in den USA nahe, der eben nicht nur von den traditionell fundamentalistischen Gruppen getragen wird.

Von Puritanismen anderer Art handelt der Aufsatz von Steven Lukes, der anhand fünf fiktiver Gesellschaftssysteme, die jeweils einer bestimmten Werthaltung »puritanisch « folgen, diskutiert, was es für Konsequenzen hat, Menschenrechte zu akzeptieren bzw. abzulehnen.

Von fundamentalistischen Tendenzen bleiben auch die modernen Wissenschaften nicht verschont. Die Sozialwissenschaften haben seit den späten 70er Jahren die Rückkehr eines $»$ Biobehavioralismus«, die Konjunktur einer »Biosoziologie« und Programme zur Erforschung der genetischen Grundlagen schwarzer Kriminalität, abweichendem Verhalten und Armut über sich ergehen lassen müssen. Seit einigen Jahren sehen sich Soziologie und Politikwissenschaft einem weiteren fundamentalen Begründungsversuch gegenüber: der Reduktion sozialer Institutionen und politischen Handelns auf das gemeinsame Fundament »rationaler Wahlhandlungen«. Der Idealtyp des Marktes wird als Urbild aller sozialen Institutionen ausgelegt. Stephan Elkins analysiert am Beispiel des Rent-Seeking-Ansatzes die implizite politische Theorie, den antipluralisti- 
schen Konservatismus einer solchen Begründungsstrategie und macht deren Inkonsistenzen deutlich, die nur durch einen unvermittelten Appell an urprotestantische Tugenden kompensiert werden können.
Der außerhalb des Heftschwerpunktes liegende Beitrag von Volker Wellhöner setzt schließlich die kritische Diskussion der herrschenden neoklassischen Theorie fort, die bereits in PROKLA 94 mit dem Artikel von Klaus Schabacker begann.

\section{WIDERSPRUEH \\ Beiträge zur sozialistischen Politik}

\section{Religion und Gewalt}

Missglückte Säkularisierung, Nationalismus, Xenophobie, Antisemitismus; Esoterik, New Age, Mythen;

Religiöser Sozialismus, Theologischer Materialismus vs. Ökonomie des Todes; Psychoanalyse, Freuds Judentum, Vaterlose Geselischaft und Gemeinschaftsgefühl

D.Claussen, H.Zinser, E.Gugenberger, P.Leuzinger, W.Spieler, H.Thielen, M. Jäger, R.Heim, M.Züfle, B.Rothschild, P.Passett, E.Modena

\section{Diskussion}

H.Schäppi: Zum SPS-Wirtschaftsprogramm R.Graf: Gramscis Gefängnishefte

Marginalien / Rezensionen / Zeitschriftenschau 